\title{
Association of posterior semicircular canal hypofunction on video-head-impulse testing with other vestibulo-cochlear deficits
}

Tarnutzer, Alexander A ; Bockisch, Christopher J ; Buffone, Elena ; Weber, Konrad P

DOI: https://doi.org/10.1016/j.clinph.2017.04.029

Posted at the Zurich Open Repository and Archive, University of Zurich ZORA URL: https://doi.org/10.5167/uzh-141358

Journal Article

Accepted Version

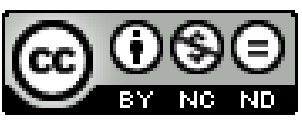

The following work is licensed under a Creative Commons: Attribution-NonCommercial-NoDerivatives 4.0 International (CC BY-NC-ND 4.0) License.

Originally published at:

Tarnutzer, Alexander A; Bockisch, Christopher J; Buffone, Elena; Weber, Konrad P (2017). Association of posterior semicircular canal hypofunction on video-head-impulse testing with other vestibulo-cochlear deficits. Clinical Neurophysiology, 128(8):1532-1541.

DOI: https://doi.org/10.1016/j.clinph.2017.04.029 
Association of posterior semicircular canal hypofunction on video-head-impulse testing with other vestibulo-cochlear deficits

Alexander A. Tarnutzer (1), Christopher J. Bockisch (1,2,3), Elena Buffone (1), Konrad P. Weber $(1,2)$

1) Department of Neurology, University Hospital Zurich and University of Zurich, Zurich Switzerland

2) Department of Ophthalmology, University Hospital Zurich and University of Zurich, Zurich Switzerland

3) Department of Otorhinolaryngology, University Hospital Zurich and University of Zurich, Zurich Switzerland

Short title: posterior canal hypofunction on video-head-impulse testing

\section{Corresponding author:}

Alexander A. Tarnutzer, MD

Department of Neurology, University Hospital Zurich

Frauenklinikstr. 26, 8091 Zurich, Switzerland

Phone: 0041442551111

Fax: 0041442554380

Email: alexander.tarnutzer@access.uzh.ch

\section{Statistics:}

word count for the text (excluding figure legends, references and abstract): 3829

word count for the abstract (limit 200 words): 200

character count for the title (including spaces): 134

number of figures: 6

number of tables: 3

supplementary files: 2

Acknowledgements: The authors thank Marco Penner for technical assistance.

Funding sources for this study: None

\section{Conflict of interest:}

Dr. Tarnutzer reports no conflict of interest

Dr. Bockisch reports no conflict of interest

Mrs. Buffone reports no conflict of interest

Dr. Weber acts as an unpaid consultant and has received funding for travel from GN Otometrics. 
Highlights:

42

43

44

45

- Isolated loss of the posterior canal on video-head-impulse testing is infrequent $(<2 \%)$.

- Loss of the posterior canal is usually associated with additional vestibulo-cochlear impairment.

- The pattern of vestibulo-cochlear impairment depends on the underlying disease. 
ABSTRACT

Objectives: The video-head-impulse test (vHIT) provides a functional assessment of all six semicircular canals (SCC). Occasionally isolated loss of the posterior canal(s) (ILPC) is diagnosed, though this finding is poorly characterized. Here we assessed how accurate that diagnosis is by measuring the co-occurrence of abnormalities on caloric irrigation, vestibularevoked myogenic-potentials and audiometry.

Methods: We identified 52 patients with ILPC (unilateral=40, bilateral=12). We determined vHIT-gains and saccade-amplitudes and correlated vHIT-findings with other vestibulocochlear tests.

Results: The most frequent diagnoses were history of vestibular neuritis (13/52), Menière's disease (12/52) and vertigo/dizziness of unclear origin (13/52). Unilateral ILPC on vHIT was accompanied by a deficient horizontal canal on calorics, saccular and/or utricular deficits ipsilesionally in 33/40 (83\%), while ipsilesional hearing-loss was noted in 24/40 (60\%). Involvement of other sensors was highest for vestibular schwannoma (100\%) and history of vestibular neuritis (92\%). Bilateral deficits in $\geq 1$ vestibulo-cochlear sensor(s) were noted in 2/12 cases with bilateral ILPC.

Conclusions: $>80 \%$ of patients with unilateral ILPC had additional deficits of other parts of the vestibular organ, while this rate was $\leq 20 \%$ for patients with bilateral ILPC.

Significance: Dizzy patients should receive testing of the posterior canals and if abnormalities are observed, additional vestibulo-cochlear testing should be obtained.

Key words: vestibular neuritis, Menière's disease, vestibular-evoked myogenic potentials, caloric irrigation, and pure tone audiogram 


\section{INTRODUCTION}

With the introduction of the video-head-impulse test (vHIT) a fast, non-invasive and quantitative assessment of all six semicircular canals (SCCs) of the vestibular organ became readily available to the clinician (Macdougall et al. , 2013). In specialized dizziness clinics, the vHIT is now a routine test for patients with dizziness, vertigo or gait imbalance. Together with caloric irrigation and otolith testing (ocular vestibular-evoked myogenic potentials (oVEMPs), cervical vestibular-evoked myogenic potentials (cVEMPs) (Curthoys, 2010, Weber et al. , 2015)), the vHIT allows comprehensive mapping of peripheral-vestibular function. As a screening test for dizzy patients, the vHIT now often provides the first evidence for peripheral-vestibular deficits. Sometimes a vHIT pattern of hypofunction restricted to one or both posterior SCCs can be observed, called isolated loss of the posterior canal (ILPC). In isolation, the clinical relevance of this finding is difficult to determine. Due to the anatomical proximity and based on clinical experience, rather a continuum between isolated involvement of a single SCC and the whole labyrinth (i.e., all 3 SCCs, both macular organs and the cochlea) is expected. These observations raise the question, to which extent other parts of the labyrinth may be involved as well in patients with seemingly isolated posterior SCC hypofunction on vHIT and what the distribution of the underlying diagnoses is. Such a closer characterization may help for the diagnostic approach to these patients. From the clinician's perspective, symptoms linked to isolated failure of the posterior SCC are often vague (typical clinical signs of unilateral peripheral-vestibular loss as spontaneous nystagmus or abnormalities on clinical head-impulse testing are frequently lacking (Chihara et al. , 2012)). Cases with isolated involvement of the posterior SCC may therefore be missed or misinterpreted as of central (e.g. stroke-related) origin (Kattah et al. , 2009).

In combination with impaired cVEMPs, unilateral hypofunction of the posterior SCC is characteristic for damage to the inferior branch of the vestibular nerve (inferior vestibulopathy, IVN) (Aw et al. , 2001, Halmagyi et al. , 2002, Chihara et al. , 2012, Kim et 
al. , 2012). ILPC may potentially be caused by various other peripheral-vestibular disorders including Menière's disease, labyrinthitis and labyrinthine concussion. Noteworthy, in most previous studies, a diagnosis of IVN was established based on caloric irrigation (bilaterally normal responses) and cVEMPs (being reduced on the affected side) (Monstad et al. , 2006, Zhang et al. , 2010, Chihara et al. , 2012, Kim et al. , 2012), while involvement of the posterior SCC was not addressed.

Here we aimed to analyze the pattern of peripheral-vestibular deficits in patients with either unilateral or bilateral ILPC on video-head-impulse testing and to correlate with underlying diagnoses. We therefore compared results from vHIT with those from other routine vestibulo-cochlear tests (caloric irrigation, ocular/cervical VEMPs, pure-tone audiogram). Potentially, this may provide patterns in vestibular hypofunction helpful in narrowing down the differential diagnosis in patients. 


\section{Material and methods}

In this retrospective study we identified 52 patients with ILPC. The protocol was approved by the Cantonal ethics commission Zurich (KEK-ZH-2013-0468). It was in accordance with the ethical standards laid down in the Declaration of Helsinki for research involving human subjects from $1964 / 2013$ ( $7^{\text {th }}$ revision). Since this was a retrospective database analysis, written informed consent from the participants could not be retrieved. This approach was in accordance with the approval from the ethics committee. Prior to analysis, patient records/information was anonymized and de-identified.

\section{Vestibular testing procedure}

'Standard vHIT procedure at the University Hospital Zurich (UHZ) requires 20 valid head-impulses for each canal (Macdougall et al. , 2013). SCCs are tested in pairs according to the planes of stimulation (horizontal plane, right-anterior-left-posterior (RALP) plane, leftanterior-right-posterior (LARP) plane). For video-oculography, we used commercially available video-head-impulse testing goggles (GN Otometrics, Taastrup, Denmark) with an infrared camera recording the right eye. Horizontal and vertical eye position was measured $(250 \mathrm{~Hz}$ frame rate) and head velocity was determined by three orthogonal gyroscopes. For further analysis, eye and head velocity values were calculated', as previously described (Tarnutzer et al. , 2016).

We reviewed saccular and utricular otolith function as assessed by air- or boneconducted cVEMPs (saccular testing) and bone-conducted oVEMPs (utricular testing). VEMPs were acquired according to the standards published elsewhere (Rosengren et al. , 2010, Weber et al. , 2015). Differences in response amplitude (left vs. right) of $>30 \%$ or absent responses were considered abnormal, i.e., indicating unilateral/bilateral hypofunction. This was based on normative values obtained with the same setup and derived cut-off values (defined as mean+2SD). Whenever air-conducted cVEMPs were inconclusive or negative, we 
obtained bone-conducted cVEMPs and judgment was based on the findings from the latter one. In cases with air-conducted cVEMP stimulation with different sound intensities, only results from the highest intensity were used.

Bithermal (i.e., warm $\left(44^{\circ} \mathrm{C}\right)$ and cold $\left(30^{\circ} \mathrm{C}\right)$ water) caloric irrigation was obtained in all patients, providing slow-phase eye velocity during stimulation. 'Unilateral hypofunction was defined as a canal paresis factor of $>25 \%$ with a preserved response on the healthy side (Halmagyi et al. , 1997), while for bilateral hypofunction a nystagmus with a mean peak slowphase velocity of $<5^{\circ} / \mathrm{sec}$ for cold- and warm-water irrigation on each side was required (Zingler et al. , 2007)', as we used previously (Tarnutzer et al. , 2016). In addition, results from pure-tone audiograms (PTA) were retrieved. We adhered to the Council on Physical Therapy - American Medical Association (CPT-AMA) guidelines for sensorineural hearingloss (Council on Physical Therapy, 1942). Therefore, hearing loss at four frequencies (500 $\mathrm{Hz}, 1 \mathrm{kHz}, 2 \mathrm{kHz}$, and $4 \mathrm{kHz}$ ) was determined and corresponding CPT-values (based on existing data taking into account the relative importance of different frequencies) were added. Total CPT values of $20 \%$ or larger were considered significant.

\section{Patient identification and statistical analysis}

All patients included here had sought medical attention because of vertigo/dizziness, gait ataxia, or hearing-loss. We searched the University Hospital Zurich vHIT database for patients with semicircular canal hypofunction that was restricted to one or both posterior canals (period: October $1^{\text {st }} 2012$ to March $21^{\text {st }}$ 2016). Eighty-eight patients (out of 2904 patients) meeting these inclusion criteria were identified for evaluation.

OtosuiteV 3.0 (GN Otometrics) was used for re-analysis of the angular vestibuloocular reflex (aVOR)-gains of the vHIT recordings. 'The gain of the aVOR was calculated as the ratio of cumulative slow-phase eye velocity over cumulative head velocity from the onset of the head impulse to the moment when head velocity returned to zero (Macdougall et al. , 
2013). For the quantification of corrective saccades we used custom-written MATLAB (The MathWorks, Natick, MA, USA) routines, providing cumulative overt saccade amplitudes (see (Weber et al. , 2009) for details)', as previously described (Tarnutzer et al. , 2016). Either a reduction in the gain of the aVOR or the occurrence of compensatory saccades was required to rate a given SCC as hypofunctional. have been proposed by The manufacturer of the videogoggles used (GN Otometrics) proposed cut-off values in aVOR-gain for the horizontal $(0.8)$ and the vertical $(0.7)$ canals. These values were in agreement with normative values for a wide range of ages reported (McGarvie et al. , 2015b). Recently proposed cut-off values suggest that saccade amplitudes above 0.7 to $0.8^{\circ} /$ trial indicate loss of function of the canal tested (MacDougall et al. , 2016, Tarnutzer et al. , 2016). Here we adhered to the cut-off value $\left(0.73^{\circ} /\right.$ trial $)$ proposed by (Tarnutzer et al., 2016$)$ as the same statistical approach was used.

All vHIT traces were independently reviewed by two neuro-otologists with extensive experience (AAT, KPW). Inter-rater agreement for individual canal function (normal vs. pathological) was 0.84 (Cohen's kappa) (Cohen, 1960). Traces were evaluated for reduced aVOR-gain, increased corrective saccades or a combination of both (Tarnutzer et al. , 2016). Discordant ratings were resolved by discussion amongst the two reviewers. Twelve patients were excluded because of more extensive peripheral-vestibular hypofunction on vHIT and 24 patients were excluded since audio-vestibular testing was incomplete. ILPC was confirmed in 52 cases. The patients' medical files were searched to retrieve the most likely diagnosis related to the ILPC along with any potential diagnosis of benign paroxysmal positional vertigo (BPPV). Menière's disease was diagnosed according to the AAO-HNS 1995 guidelines (1995). The diagnosis of vestibular neuritis was based on clinical grounds (defined as a single episode with acute-onset, prolonged vertigo or dizziness and spontaneous nystagmus) as documented in the patient's medical records and - if available - on vestibular testing in the acute stage. MR-imaging was required to confirm vestibular schwannoma. 
We used the generalized linear model (GLM) implemented in SPSS 22 (IBM, 185 Armonk, NY, USA) for statistical analysis and applied Fisher's least significant difference 186 (LSD) method to compensate for multiple comparisons. Note that we kept the level of 187 significance always at a p-value of 0.05 . 


\section{Results}

Fifty-two cases with unilateral $(n=40)$ or bilateral $(n=12)$ ILPC were included (22 females, $59.5 \pm 15.4$ years old, mean \pm 1 standard deviation (SD). From the 12 bilateral cases, five were previously reported in a different study (Tarnutzer et al. 2016). The most frequent causes of unilateral ILPC were past medical history of VN (13/40, 33\%), Menière's disease $(10 / 40,25 \%)$ and vestibular schwannoma (7/40, 18\%) (Table 1 and supplementary file 1$)$. In case of bilateral ILPC, the diagnosis remained unclear in the majority of cases $(7 / 12,58 \%)$. A typical example of left-sided ILPC due to vestibular schwannoma is shown in Figure 1.

$$
\text { /* Figure } 1 \text { about here */ }
$$

\section{vHIT - gains and cumulative saccade amplitudes}

Gain values are shown separately for cases with left-sided (Fig. 2A, n=24), right-sided (Fig 2B, n=16) and bilateral (Fig. 2C, n=12) ILPC. While for the anterior and horizontal gains were well above the cut-off, they were decreased (i.e., below the cut-off of 0.7 ) for the ipsilesional posterior SCC. Likewise, cumulative saccade amplitudes were increased for all three subgroups (Fig 2, panels D-F) for the affected posterior SCC(s), being clearly above the predefined cut-off of $0.73^{\circ} /$ trial.

/* Figure 2 about here */ 

in $26 / 40$ cases $(65 \%$; asymmetry ratio $=65 \pm 15 \%($ mean $\pm 1 \mathrm{SD}))$ with unilateral ILPC (Fig. 3$)$. In three unilateral cases caloric irrigation showed impairment on the side opposite to the ILPC. From the 12 cases with bilateral ILPC on vHIT, caloric irrigation was bilaterally absent in $1(8 \%)$ and unilaterally impaired in $4(33 \%)$ (supplementary file 2). Amongst the different diagnoses, the rate of ipsilesional impairment on caloric irrigation varied considerably, being

221

/* Figure 3 about here */

\section{oVEMPs and cVEMPs}

oVEMPs were ipsilesionally abnormal (i.e., asymmetry ratio $>30 \%$ ) in 20/40 cases $(50 \%$; asymmetry ratio $=60 \pm 23 \%)$ with unilateral ILPC (Fig. 4). This included six cases with bilaterally absent responses on oVEMPs. Significant contralesional impairment was noted in one case. In patients with bilateral ILPC, no cases with bilaterally absent oVEMPs were noted, while oVEMPs were unilaterally impaired in two cases (supplementary file 2). Rates of ipsilesional utricular involvement were highest for vestibular schwannoma (7/7), past medical history of $\mathrm{VN}(7 / 13)$ and various causes (2/4), while being infrequent in Menière's disease and unclear (unilateral/bilateral) cases (Tables 2 and 3).

Significant ipsilesional impairment of cVEMPs (i.e., asymmetry ratio $>30 \%$ ) was noted in $16 / 40$ cases $(40 \%$; asymmetry ratio $=72 \pm 29 \%)$ with unilateral ILPC (Figure 5). For bilateral cases, both unilaterally reduced (3/12) and bilaterally absent (1/12) cVEMPs were noted (supplementary file 2). Amongst the different diagnoses, the fraction of saccular 

medical history of VN (7/13).

\section{Pure tone audiogram} was noted in $4 / 52$ cases $(8 \%)$.

involvement on the side of ILPC was highest for vestibular schwannoma (6/7) and past

$$
\text { /* Figures } 4 \text { and } 5 \text { about here */ }
$$

Significant hearing-loss was identified in $32 / 52$ cases (see supplementary file 2). While hearing-loss was restricted to the side with ILPC in 18/40 (45\%) unilateral cases, it was bilateral in $6 / 40(15 \%)$ patients. Amongst patients with bilateral ILPC, hearing-loss was unilateral in five patients and bilateral in one patient.

\section{Extent of additional vestibulo-cochlear lesions}

Involvement of at least one additional part of the vestibular organ ipsilesional was noted in 33/40 cases with unilateral ILPC (83\%) (Table 2). Bilateral involvement of at least one vestibular sensor was found in 2/12 (17\%) cases with bilateral ILPC. A lesion pattern consistent with ipsilesional involvement of the superior branch of the vestibular nerve (impaired oVEMPs and caloric irrigation) was found in 15/40 cases (38\%) with unilateral ILPC. None of the cases with bilateral ILPC presented with such a pattern bilaterally.

A lesion pattern consistent with ipsilesional involvement of the inferior branch of the vestibular nerve (impaired cVEMPs and hearing-loss) was noted in 14/40 (35\%) cases with unilateral ILPC, while none of the cases with bilateral ILPC presented with such a pattern bilaterally. Loss of function on all vestibulo-cochlear tests (caloric irrigation, oVEMPs, cVEMPs, pure-tone audiometry) ipsilesionally was found in 8 cases with unilateral ILPC (past medical history of $\mathrm{VN}=3$; schwannoma $=5$ ), while completely normal audio-vestibular 
In cases with unilateral ILPC, the number of affected sensors ipsilesionally depended

on the diagnosis, being higher for vestibular schwannoma than for all other diagnoses $(\mathrm{p} \leq 0.006$, generalized linear model) (Fig. 6). While all sensors were affected with a similar frequency for the vestibular schwannoma cases, cochlear loss of function was most frequent in Menière's disease and impairment of the horizontal SCC on caloric irrigation in past medical history of VN and various diagnoses.

$$
\text { /* Figure } 6 \text { about here */ }
$$

\section{Benign paroxysmal positional vertigo (BPPV) in ILPC}

With partial impairment of the SCCs and the otolith organs, risk for BPPV is increased (Karlberg et al. , 2000). In our cohort three patients with unilateral ILPC had posterior-canal BPPV ipsilesionally and one patient with bilateral ILPC had posterior-canal BPPV unilaterally. In two patients BPPV was reported on the healthy side. Diagnoses amongst these six patients were past medical history of $\mathrm{VN}(\mathrm{n}=1)$, various (history of traumatic brain injury $(n=2))$, Menière's disease $(n=1)$ and unclear unilateral $(n=1)$ or bilateral $(n=1)$ causes. 


\section{Discussion}

Based on the video-head-impulse test, we identified isolated unilateral or bilateral hypofunction of the posterior SCCs in 52 out of 2904 patients (1.8\%), making it an infrequent, but regular finding. However, more extensive vestibular testing revealed additional ipsilesional peripheral-vestibular deficits in $83 \%$ of patients with unilateral ILPC. In those patients with bilateral ILPC, additional peripheral-vestibular deficits were rarely bilateral (17\%), but more often unilateral (42\%). These findings suggest that truly isolated posterior SCC-loss is rather the exception than the rule and emphasizes the need for more extensive vestibulo-cochlear testing in these patients. The pattern of involvement of other vestibulocochlear sensors was diagnosis-dependent, being most widespread in patients with past medical history of $\mathrm{VN}$ and vestibular schwannoma. This was in contrast to patients with ILPC of unclear origin, showing less vestibulo-cochlear involvement, guiding to a different diagnosis.

Our case series included one patient with bilateral ILPC and a history of brainstem encephalitis. Damage to the medial longitudinal fasciculus (MLF) on both sides may explain the ILPC in this patient, as previously described by Cremer and co-workers in patients with multiple scleroris and internuclear ophthalmoplegia (Cremer et al. , 1999).

\section{Patterns of peripheral vestibulo-cochlear hypofunction in ILPC}

While all our patients were selected based on a normal horizontal video-head-impulse test, nonetheless most showed abnormal caloric responses (ipsilesional impairment in $65 \%$ of patients with unilateral ILPC; unilateral impairment in 17\% of patients with bilateral ILPC). Furthermore, unilateral ILPC was accompanied by ipsilesional loss of other vestibulocochlear sensors supplied by the inferior branch (hearing-loss and saccular hypofunction) in only $35 \%$ of cases. This suggests that either in the majority of cases the underlying 
pathomechanism was not damage to the inferior branch of the vestibular nerve (or the vestibular artery) or that damage within this branch was only partial.

\section{Past medical history of $V N$}

Since VN is related to damage of the vestibular nerve or its branches, the typical pattern of vestibular hypofunction can be predicted (Curthoys 2009). As the posterior SCC is innervated by the inferior branch, additional deficits of the sacculus are expected. From the 13 cases with past medical history of $\mathrm{VN}$, additional hypofunction of at least one vestibular sensor innervated by the superior branch was noted in ten (77\%). This suggests that these cases likely had involvement of both branches ("combined VN"), while only three cases showed damage restricted to sensors supplied by the inferior branch. In the medical files of seven of our patients with past medical history of $\mathrm{VN}$ we found results from previous quantitative head-impulse-testing, being recorded within days after symptom-onset in five out of seven patients. These remote measurements demonstrated previous involvement of the superior branch (mostly the horizontal SCC) in all seven cases. This suggests that at least in these seven patients (and possibly in the other six patients with past medical history of $\mathrm{VN}$ as well) horizontal SCC-function had recovered while the posterior SCC remained defective. A detailed patient interview will readily identify a past medical history of $\mathrm{VN}$ in these cases.

Noteworthy, we cannot provide any estimates on how frequent such partial recovery after VN is, as ILPC was a prerequisite for inclusion. Previously, other studies comparing recovery after unilateral $\mathrm{VN}$ using $\mathrm{vHIT}$ and caloric irrigation demonstrated discrepancies (Mahringer et al. , 2014, Zellhuber et al. , 2014, Patel et al. , 2016). In a population of dizzy patients with unilateral hypofunction of the horizontal SCC on caloric irrigation, vHIT was abnormal in only 41\% (Mahringer et al. , 2014), with numbers being highest in the acute stage and decreasing with increasing disease duration. Such discrepancies for horizontal SCCfunction may be related to the fact that unlike for the (video)-head-impulse test - applying a 
physiological stimulus (i.e., fast head-turns, providing bilateral stimulation) - vestibular stimulation in case of caloric irrigation is artificial (and unilateral) and lacks the high frequencies provided in the vHIT. In a recent study focusing on patients with sudden sensorineural hearing-loss and acute vertigo, posterior-canal hypofunction was found in $74 \%$ of patients, while other vestibular sensors were affected about one third of cases (Pogson et al. ,2016). Most patients in this study received a diagnosis of labyrinthitis.

\section{Menière's disease}

In bilateral Menière's disease, we have found relative sparing of the anterior canals (Tarnutzer et al. , 2016). Unlike VN, the distribution of affected vestibulo-cochlear sensors does not follow a specific pattern linked to the innervation or vascular supply in Menière's disease, allowing virtually any combination of impairment. In our Menière's disease subgroup, accompanying ipsilesional hearing-loss was the most consistent finding in cases with unilateral ILPC (90\%), followed by a reduced ipsilesional response on caloric irrigation in $50 \%$. Involvement of the otolith organs was infrequent (oVEMPs $=20 \%$; cVEMPs $=30 \%$ ). Dissociations between video-head-impulse testing and caloric irrigation of the horizontal SCCs have been linked to the altered anatomy of the hydropic labyrinth and subsequently reduced responses of the horizontal SCC to caloric irrigation, but not to head-impulse testing (McGarvie et al. , 2015a). This, however, does not explain the pattern of reduced posterior SCC-function with preserved anterior and horizontal canals on video-head-impulse testing.

\section{Vestibular Schwannoma}

We found seven cases of ILPC from vestibular nerve compression due to schwannoma. While for small $(\leq 14 \mathrm{~mm}$ diameter) schwannoma at least one (out of five) vestibular test was abnormal in 59\%, this number increased to at least three abnormal tests in 
$86 \%$ of patients with medium-to-large schwannoma ( $>14 \mathrm{~mm})$ (Taylor et al. , 2015).

Vestibular schwannoma will either originate from the superior or the inferior branch and growth and compression results in hypofunction of sensors linked to either branch in the majority of cases (58\%) (Taylor et al. , 2015). Interestingly, we identified seven patients who showed sparing of the anterior and horizontal SCC on video-head-impulse testing. A dissociation between horizontal SCC-function on caloric irrigation (being impaired) and video-head-impulse testing (being preserved) was previously reported in a case series (Blodow et al. , 2015) and in a single case with intra-labyrinthine schwannoma (Machner et al. , 2007). Due to our study design, we cannot provide any data on the frequency of this pattern amongst all patients with vestibular schwannoma. Considering hypofunction on caloric irrigation and on both cVEMPs and oVEMPs in five out of seven cases, however, deficits were extensive in our cases as well, despite sparing of the anterior and horizontal canal on the vHIT.

\section{Low rate of involvement of other vestibular sensors in unclear cases}

Cases with unclear cause of unilateral/bilateral ILPC were distinct from the other subgroups in several aspects: 1) regarding leading symptoms, (episodic) gait imbalance was more frequent than vertigo or dizziness, 2 ) other vestibular sensors were affected in $50 \%$ or less of cases, indicating more limited vestibulo-cochlear deficits than in other subgroups, and 3) ipsilesional hearing-loss was rarely noted. Likely failure to identify a specific cause was linked to the more subtle clinical presentation and negative vestibulo-cochlear testing in these patients. On video-head-impulse testing, however, ILPC was clearly detectable in these cases and average gains and cumulative saccade amplitudes of the posterior $\mathrm{SCC}(\mathrm{s})$ were not significantly different from the other subgroups. Within the spectrum of clinical presentations of bilateral vestibular loss, those cases with bilateral ILPC may represent the mildest form 
with sparing of the other SCCs. This contrasts cases with more extensive bilateral vestibular impairment such as due to inner-ear infections or gentamicin (Tarnutzer et al. , 2016).

\section{Limitations}

We used a retrospective study design and selected a subgroup of patients presenting to our clinic based on the results of a single test. We therefore cannot make any conclusions on the prevalence of ILPC compared to other patterns of peripheral-vestibular hypofunction. With a clear focus on vertigo and dizziness, auditory testing was less detailed than vestibular testing. We could therefore not retrieve more detailed information about auditory nerve function than pure tone audiograms.

While vestibular testing was standardized and all head-impulses were analyzed with the same version of the GN-Otometrics software, we did not perform a uniform clinical examination and therefore had to rely on the diagnosis as noted in the medical files. Noteworthy, all patients were seen at the interdisciplinary center for dizziness and balance disorders by specialized neuro-otologists. While for Menière's disease AAO-HNS 1995 guidelines were used routinely and MR-imaging was mandatory for cases with vestibular schwannoma, the diagnosis of vestibular neuritis was based on clinical grounds. In 13/52 patients no specific diagnosis was provided in the medical files available. Potentially, repeated and structured history taking and detailed vestibulo-cochlear testing may have resulted in a diagnosis. Furthermore, we might have missed follow-up diagnostic testing performed at another hospital, not documented in our files. 
Overall, ILPC is an infrequent finding, representing less than $2 \%$ of all patients having 404 received video-head-impulse testing at our institution. In patients presenting with dizziness, 405 the posterior canals should be measured and if abnormalities are observed, more extensive 406 vestibulo-cochlear testing should be obtained. In these patients, additional vestibular deficits 407 can be expected in more than $80 \%$. The pattern of vestibulo-cochlear impairment depends on 408 the underlying disease, being highest for vestibular schwannoma and past medical history of 409 VN. 
TABLES

Table 1: isolated loss of the posterior canal(s) on vHIT - underlying diagnoses

\begin{tabular}{|l|l|l|l|}
\hline Diagnosis & Unilateral ILPC (\%) & Bilateral ILPC (\%) & Total \\
\hline Hypofunction of unclear origin & $6(12)$ & $7(13)$ & $13(25)$ \\
\hline Past medical history of VN & $13(25)$ & $0(0)$ & $13(25)$ \\
\hline Menière's disease & $10(19)$ & $2(4)$ & $12(23)$ \\
\hline Vestibular schwannoma & $7(13)$ & $0(0)$ & $7(13)$ \\
\hline Possible vestibular migraine & $1(2)$ & $1(2)$ & $2(4)$ \\
\hline History of TBI & $1(2)$ & $1(2)$ & $2(4)$ \\
\hline Acute VN & $1(2)$ & $0(0)$ & $1(2)$ \\
\hline Repetitive hemorrhagic labyrinthitis & $1(2)$ & $0(0)$ & $1(2)$ \\
\hline History of brainstem encephalitis & $0(0)$ & $1(2)$ & $1(2)$ \\
\hline All & $\mathbf{4 0 ( 7 7 \% )}$ & $\mathbf{1 2}(\mathbf{2 3} \%)$ & $\mathbf{5 2}(\mathbf{1 0 0} \%)$ \\
\hline
\end{tabular}

Abbreviations: ILPC $=$ isolated loss of the posterior canal(s); TBI=traumatic brain injury; $\mathrm{VN}=$ vestibular neuritis. 


\begin{tabular}{|c|c|c|c|c|c|c|c|}
\hline Disease & $\begin{array}{l}\text { Caloric irrigation: } \\
\text { Impaired } \\
\text { horizontal canal (n, } \\
\%)^{*}\end{array}$ & $\begin{array}{l}\text { oVEMPs: } \\
\text { Impaired } \\
\text { utriculus (n, } \\
\%) \dagger\end{array}$ & $\frac{\text { Calorics \& oVEMPs: }}{\text { both impaired }(n, \%)}$ & $\begin{array}{l}\text { cVEMP: } \\
\text { impaired } \\
\text { sacculus }(n, \%) \dagger\end{array}$ & $\begin{array}{l}\text { Impairment of any of the } \\
\text { three (calorics, oVEMP, } \\
\underline{\text { cVEMP) }(n, \%)}\end{array}$ & $\begin{array}{l}\text { PTA: } \\
\text { Hearing loss (CPT- } \\
\text { AMA) }(n, \%) \$\end{array}$ & $\begin{array}{l}\text { PTA \& cVEMPs: } \\
\text { Both impaired (n, } \\
\%)\end{array}$ \\
\hline $\begin{array}{l}\text { Past medical } \\
\text { history of VN } \\
(n=13)\end{array}$ & $9,69 \%$ & $7,53.8 \%$ & $6,46 \%$ & $7,54 \%$ & $12,92 \%$ & $5,39 \%$ & $4,31 \%$ \\
\hline $\begin{array}{l}\text { Menière's } \\
\text { disease } \\
(\mathbf{n}=\mathbf{1 0})\end{array}$ & $5,50 \%$ & $2,20.0 \%$ & $2,20 \%$ & $3,30 \%$ & $7,70 \%$ & $9,90 \%$ & $3,30 \%$ \\
\hline $\begin{array}{l}\text { Schwannoma } \\
(n=7)\end{array}$ & $6,86 \%$ & $7,100.0 \%$ & $6,87 \%$ & $6,87 \%$ & $7,100 \%$ & $7,100 \%$ & $6,87 \%$ \\
\hline $\begin{array}{l}\text { Unilateral } \\
\text { ILPC of } \\
\text { unclear origin } \\
(n=6)\end{array}$ & $2,33 \%$ & $2,33.3 \%$ & $1,17 \%$ & $0,0 \%$ & $3,50 \%$ & $1,17 \%$ & $0,0 \%$ \\
\hline $\begin{array}{l}\text { Various causes } \\
(n=4) \S\end{array}$ & $4,100 \%$ & $2,50.0 \%$ & $2,50 \%$ & $0,0 \%$ & $4,100 \%$ & $2,50 \%$ & $1,25 \%$ \\
\hline $\begin{array}{l}\text { Total unilat. } \\
\text { ILPC }(n=40)\end{array}$ & $\begin{array}{l}26 \\
(65 \%)\end{array}$ & $\begin{array}{l}20 \\
(50 \%)\end{array}$ & $\begin{array}{l}15 \\
(38 \%)\end{array}$ & $\begin{array}{l}16 \\
(40 \%)\end{array}$ & $\begin{array}{l}33 \\
(83 \%)\end{array}$ & $\begin{array}{l}24 \\
(60 \%)\end{array}$ & $\begin{array}{l}14 \\
(35 \%)\end{array}$ \\
\hline
\end{tabular}

* Impairment of the horizontal canal was considered significant if the asymmetry ratio was $>25 \%$ in favor of the other side or if mean cold/warm water caloric irrigation peak slow-phase velocity on both sides was less than $5 \%$ sec (Zingler et al. , 2007).

$\dagger$ Impairment of vestibular-evoked myogenic potentials was considered significant if the asymmetry ratio was $>30 \%$ in favor of the opposite side or if responses were bilaterally absent.

t Significant hearing-loss was defined as CPT-AMA values $>20 \%$.

$\S$ Included diagnoses are: possible vestibular migraine $(n=1)$, history of traumatic brain injury $(n=1)$, acute VN $(n=1)$, repetitive hemorrhagic labyrinthitis $(n=1)$. 


\begin{tabular}{|c|c|c|c|c|c|c|c|}
\hline Disease & $\begin{array}{l}\text { Caloric irrigation: } \\
\text { Impaired } \\
\text { horizontal canal (n, } \\
\%) \dagger\end{array}$ & $\begin{array}{l}\text { oVEMPs: } \\
\text { Impaired } \\
\text { utriculus (n, } \\
\%) \ddagger\end{array}$ & $\begin{array}{l}\text { Calorics \& oVEMPs: } \\
\text { both impaired (n, \%) }\end{array}$ & $\begin{array}{l}\text { cVEMP: } \\
\text { impaired } \\
\text { sacculus }(n, \%)\end{array}$ & $\begin{array}{l}\text { Impairment of any of the } \\
\text { three (calorics, oVEMP, } \\
\text { cVEMP) (n, \%) }\end{array}$ & $\begin{array}{l}\text { PTA: } \\
\text { Hearing loss (CPT- } \\
\text { AMA) }(n, \%) \S\end{array}$ & $\begin{array}{l}\text { PTA \& cVEMPs: } \\
\text { Both impaired (n, } \\
\%)\end{array}$ \\
\hline $\begin{array}{l}\text { Menière's } \\
\text { disease } \\
(\mathbf{n}=\mathbf{2})\end{array}$ & $0(+2), 0 \%(100 \%)$ & $0(+0), 0 \%(0 \%)$ & $0(+0), 0 \%(0 \%)$ & $0(+1), 0 \%(50 \%)$ & $0(+2), 0 \%(100 \%)$ & $0(+1), 0 \%(50 \%)$ & $0(+1), 0 \%(50 \%)$ \\
\hline $\begin{array}{l}\text { Bilateral ILPC } \\
\text { of unclear } \\
\text { origin } \\
(n=7)\end{array}$ & $1(+0), 14 \%(0 \%)$ & $0(+1), 0 \%(14 \%)$ & $0(+1), 0 \%(14 \%)$ & $1(+2), 14 \%(29 \%)$ & $2(+1), 29 \%(14 \%)$ & $1(+3), 14 \%(57 \%)$ & $0(+2), 0 \%(29 \%)$ \\
\hline $\begin{array}{l}\text { Various causes } \\
(n=3) \|\end{array}$ & $0(+2), 0 \%(67 \%)$ & $0(+1), 0 \%(33 \%)$ & $0(+1), 0 \%(33 \%)$ & $0(+0), 0 \%(0 \%)$ & $0(+2), 0 \%(67 \%)$ & $0(+1), 0 \%(33 \%)$ & $0(+0), 0 \%(0 \%)$ \\
\hline $\begin{array}{l}\text { Total bilateral } \\
\text { ILPC }(n=12)\end{array}$ & $1(+4), 8 \%(33 \%)$ & $\begin{array}{l}0(+2), 0 \% \\
(17 \%)\end{array}$ & $0(+2), 0 \%(17 \%)$ & $1(+3), 0 \%(25 \%)$ & $2(+5), 17 \%(42 \%)$ & $1(+5), 8 \%(42 \%)$ & $0(+3), 0 \%(25 \%)$ \\
\hline
\end{tabular}

* Cases with unilateral hypofunction but bilateral ILPC are in brackets.

$\dagger$ Impairment of the horizontal canal was considered significant if the asymmetry ratio was $>25 \%$ in favor of the other side or if mean cold/warm water caloric irrigation mean peak slow-phase velocity on both sides was less than $5 \%$ sec (Zingler et al. , 2007).

$\$$ Impairment of vestibular-evoked myogenic potentials was considered significant if the asymmetry ratio was $>30 \%$ in favor of the opposite side or if responses were bilaterally absent.

$\S$ Significant hearing-loss was defined as CPT-AMA values $>20 \%$.

|| Included diagnoses are: possible vestibular migraine $(n=1)$, history of traumatic brain injury $(n=1)$ and history of brainstem encephalitis $(n=1)$. 


\section{FIGURE LEGENDS}

437

438

439

440

441

442

443

444

445

446

447

448

449

450

451

452

453

454

455

456

457

458

459

\section{Figure 1:}

Illustrative example of a patient with unilateral isolated loss of the left posterior SCC on video-head-impulse testing and as indicated by decreased gain (0.54) and increased covert and overt catch-up saccades (black arrow) (panel A). He was diagnosed with left-sided vestibular schwannoma (panel B: axial contrast-enhanced MR-image). Additional vestibular testing revealed ipsilesionally reduced response of the horizontal SCC on caloric irrigation (panel C; asymmetry ratio $=44 \%$ ) and impaired saccular (panel D; asymmetry-ratio $=51 \%$ ) and utricular (panel E; asymmetry ratio=47\%) function. Furthermore, there was moderate leftsided sensorineural hearing-loss for frequencies above $2 \mathrm{kHz}$ on pure-tone audiogram (panel F).

\section{Figure 2:}

Mean $( \pm 1 \mathrm{SD})$ gains (panels A-C) and cumulative saccadic amplitudes (panels D-F) of all patients $(n=52)$ shown separately depending on the side of posterior canal hypofunction (left, right, bilateral). Gain values (from 0 to 1$)$ and cumulative saccadic amplitudes ( $\% /$ trial, from 0 to 2$)$ are provided along the different hexagons. Cut-off values for reduced gains $(<0.8$ for the horizontal canals, $<0.7$ for the vertical canals) and for abnormally increased cumulative saccade amplitudes $\left(>0.73^{\circ} /\right.$ trial $)$ are indicated by dashed lines.

\section{Figure 3:}

Asymmetry ratios (AR; individual values and mean $\pm 1 \mathrm{SD})$ for caloric irrigation are shown both for all patients with unilateral ILPC and the different diagnoses separately. Asymmetry ratios were considered significant if larger than $\pm 25 \%$, as indicated by the dashed vertical 
lines and the light grey-shaded background. Cases were separated for each group based on the lesion pattern on caloric irrigation compared to the hypofunction identified on video-headimpulse testing. We distinguished between cases with weaker responses on caloric irrigation on the same side as the ILPC on vHIT (black symbols, AR with a positive sign, shown on the right side, and cases with weaker response on caloric irrigation on the opposite side as the ILPC (grey symbols, AR with a negative sign, shown on the left side). Note that the 12 cases with bilateral ILPC are not shown here (see Figure 4).

\section{Figure 4:}

Asymmetry ratios (AR; individual values and mean $\pm 1 \mathrm{SD}$ ) for oVEMPs are shown for all patients with unilateral ILPC and the different underlying diagnoses separately. AR were considered significant if larger than $\pm 30 \%$, as indicated by the dashed vertical lines and the light grey-shaded background. Cases were separated for each group based on the lesion pattern in oVEMPs compared to the hypofunction identified on the video-head-impulse test. We distinguished between cases with weaker responses on oVEMPs on the same side as the ILPC on vHIT (black symbols, AR with a positive sign, shown on the right side,) and cases with weaker response on oVEMPs on the opposite side as the ILPC (grey symbols, AR with a negative sign, shown on the left side). Note that six patients had bilaterally absent oVEMPs (not shown). Those 12 cases with bilateral ILPC are not shown here (see supplementary file 2).

\section{Figure 5:}

Asymmetry ratios (AR; individual values and mean $\pm 1 \mathrm{SD}$ ) for cVEMPs are shown for all patients with unilateral ILPC and the different underlying diagnoses separately. AR were considered significant if larger than $\pm 30 \%$, as indicated by the dashed vertical lines and the light grey-shaded background. Cases were separated for each group based on the lesion 
pattern in cVEMPs compared to the hypofunction identified on the video-head-impulse test.

487

488

489

490

491

492

493

494

495

496

497

498

499

500

501

502

503

We distinguished between cases with weaker responses on cVEMPs on the same side as the ILPC on vHIT (black symbols, AR with a positive sign, shown on the right side,) and cases with weaker response on cVEMPs on the opposite side as the ILPC (grey symbols, AR with a negative sign, shown on the left side). Note that one patient hat bilaterally absent cVEMPs (not shown). Those 12 cases with bilateral ILPC are not shown here (see supplementary file 2).

\section{Figure 6:}

Bar plot illustrating mean $( \pm 1 \mathrm{SD})$ numbers of other ipsilesionally impaired vestibulo-cochlear sensors for different causes of unilateral ILPC $(n=40)$. Each bar represents the relative frequency of hypofunction for the different sensors tested (hearing-loss due to cochlear loss of function, abnormal horizontal SCC on caloric irrigation, reduced saccular response on cVEMPs, reduced utricular response on oVEMPs) for a given cause in a stacked fashion (see inset for further explanations). On the right side, average values for all subjects are shown. Statistically significant differences (generalized linear model, least significant difference (LSD) for compensation of multiple tests) are indicated by an asterisk ('*'). Due to small numbers no such analysis was performed for the 12 cases with bilateral ILPC. 
505 Committee on Hearing and Equilibrium guidelines for the diagnosis and evaluation of therapy in Meniere's disease. American Academy of Otolaryngology-Head and Neck Foundation, Inc. Otolaryngol Head Neck Surg. 1995;113:181-5.

508 509

Aw ST, Fetter M, Cremer PD, Karlberg M, Halmagyi GM. Individual semicircular canal function in superior and inferior vestibular neuritis. Neurology. 2001;57:768-74.

Blodow A, Blodow J, Bloching MB, Helbig R, Walther LE. Horizontal VOR function shows frequency dynamics in vestibular schwannoma. Eur Arch Otorhinolaryngol. 2015;272:21438.

Chihara Y, Iwasaki S, Murofushi T, Yagi M, Inoue A, Fujimoto C, et al. Clinical characteristics of inferior vestibular neuritis. Acta Otolaryngol. 2012;132:1288-94.

Cohen J. A coefficient for agreement for nominal scales. Educ Psychol Meas. 1960;20:37-46.

Council on Physical Therapy AMA. Tentative standard procedures for evaluating the percentage of useful hearing loss in medicolegal cases. JAMA. 1942;119:1108-9.

Cremer PD, Migliaccio AA, Halmagyi GM, Curthoys IS. Vestibulo-ocular reflex pathways in internuclear ophthalmoplegia. Ann Neurol. 1999;45:529-33.

Curthoys IS. A critical review of the neurophysiological evidence underlying clinical vestibular testing using sound, vibration and galvanic stimuli. Clin Neurophysiol. 2010;121:132-44.

Halmagyi GM, Aw ST, Karlberg M, Curthoys IS, Todd MJ. Inferior vestibular neuritis. Ann N Y Acad Sci. 2002;956:306-13. 
Halmagyi GM, Yavor RA, McGarvie LA. Testing the vestibulo-ocular reflex. In: Alford BR, Jerger J, Jenkins HA, editors. Electrophysiologic Evaluation in Otolaryngology. Basel: Karger; 1997. p. 132-54.

Karlberg M, Hall K, Quickert N, Hinson J, Halmagyi GM. What inner ear diseases cause benign paroxysmal positional vertigo? Acta Otolaryngol. 2000;120:380-5.

Kattah JC, Talkad AV, Wang DZ, Hsieh YH, Newman-Toker DE. HINTS to diagnose stroke in the acute vestibular syndrome: three-step bedside oculomotor examination more sensitive than early MRI diffusion-weighted imaging. Stroke. 2009;40:3504-10.

Kim JS, Kim HJ. Inferior vestibular neuritis. J Neurol. 2012;259:1553-60.

Macdougall HG, McGarvie LA, Halmagyi GM, Curthoys IS, Weber KP. The video Head Impulse Test (vHIT) detects vertical semicircular canal dysfunction. PLoS One. 2013;8:e61488.

MacDougall HG, McGarvie LA, Halmagyi GM, Rogers SJ, Manzari L, Burgess AM, et al. A new saccadic indicator of peripheral vestibular function based on the video head impulse test. Neurology. 2016;87:410-8.

Machner B, Gottschalk S, Sander T, Helmchen C, Rambold H. Intralabyrinthine schwannoma affecting the low but not high frequency function of the vestibulo-ocular reflex: implications for the clinical diagnosis of chronic peripheral vestibular deficits. J Neurol Neurosurg Psychiatry. 2007;78:772-4.

Mahringer A, Rambold HA. Caloric test and video-head-impulse: a study of vertigo/dizziness patients in a community hospital. Eur Arch Otorhinolaryngol. 2014;271:463-72. 
McGarvie LA, Curthoys IS, MacDougall HG, Halmagyi GM. What does the dissociation between the results of video head impulse versus caloric testing reveal about the vestibular dysfunction in Meniere's disease? Acta Otolaryngol. 2015a;135:859-65.

McGarvie LA, MacDougall HG, Halmagyi GM, Burgess AM, Weber KP, Curthoys IS. The Video Head Impulse Test (vHIT) of Semicircular Canal Function - Age-Dependent Normative Values of VOR Gain in Healthy Subjects. Front Neurol. 2015b;6:154.

Monstad P, Okstad S, Mygland A. Inferior vestibular neuritis: 3 cases with clinical features of acute vestibular neuritis, normal calorics but indications of saccular failure. BMC Neurol. $2006 ; 6: 45$.

Patel M, Arshad Q, Roberts RE, Ahmad H, Bronstein AM. Chronic Symptoms After Vestibular Neuritis and the High-Velocity Vestibulo-Ocular Reflex. Otol Neurotol. 2016;37:179-84.

Pogson JM, Taylor RL, Young AS, McGarvie LA, Flanagan S, Halmagyi GM, et al. Vertigo with sudden hearing loss: audio-vestibular characteristics. J Neurol. 2016;263:2086-96.

Rosengren SM, Welgampola MS, Colebatch JG. Vestibular evoked myogenic potentials: past, present and future. Clin Neurophysiol. 2010;121:636-51.

Tarnutzer AA, Bockisch CJ, Buffone E, Weiler S, Bachmann LM, Weber KP. Diseasespecific sparing of the anterior semicircular canals in bilateral vestibulopathy. Clin Neurophysiol. 2016;127:2791-801.

Taylor RL, Kong J, Flanagan S, Pogson J, Croxson G, Pohl D, et al. Prevalence of vestibular dysfunction in patients with vestibular schwannoma using video head-impulses and vestibular-evoked potentials. J Neurol. 2015;262:1228-37. 
568 Weber KP, Aw ST, Todd MJ, McGarvie LA, Curthoys IS, Halmagyi GM. Horizontal head 569 impulse test detects gentamicin vestibulotoxicity. Neurology. 2009;72:1417-24.

570 Weber KP, Rosengren SM. Clinical utility of ocular vestibular-evoked myogenic potentials 571 (oVEMPs). Curr Neurol Neurosci Rep. 2015;15:22.

572 Zellhuber S, Mahringer A, Rambold HA. Relation of video-head-impulse test and caloric 573 irrigation: a study on the recovery in unilateral vestibular neuritis. Eur Arch Otorhinolaryngol. $574 \quad 2014 ; 271: 2375-83$.

575 Zhang D, Fan Z, Han Y, Yu G, Wang H. Inferior vestibular neuritis: a novel subtype of 576 vestibular neuritis. J Laryngol Otol. 2010;124:477-81.

577 Zingler VC, Cnyrim C, Jahn K, Weintz E, Fernbacher J, Frenzel C, et al. Causative factors 578 and epidemiology of bilateral vestibulopathy in 255 patients. Ann Neurol. 2007;61:524-32.

579 


\section{A \\ Video-head-impulse testing}
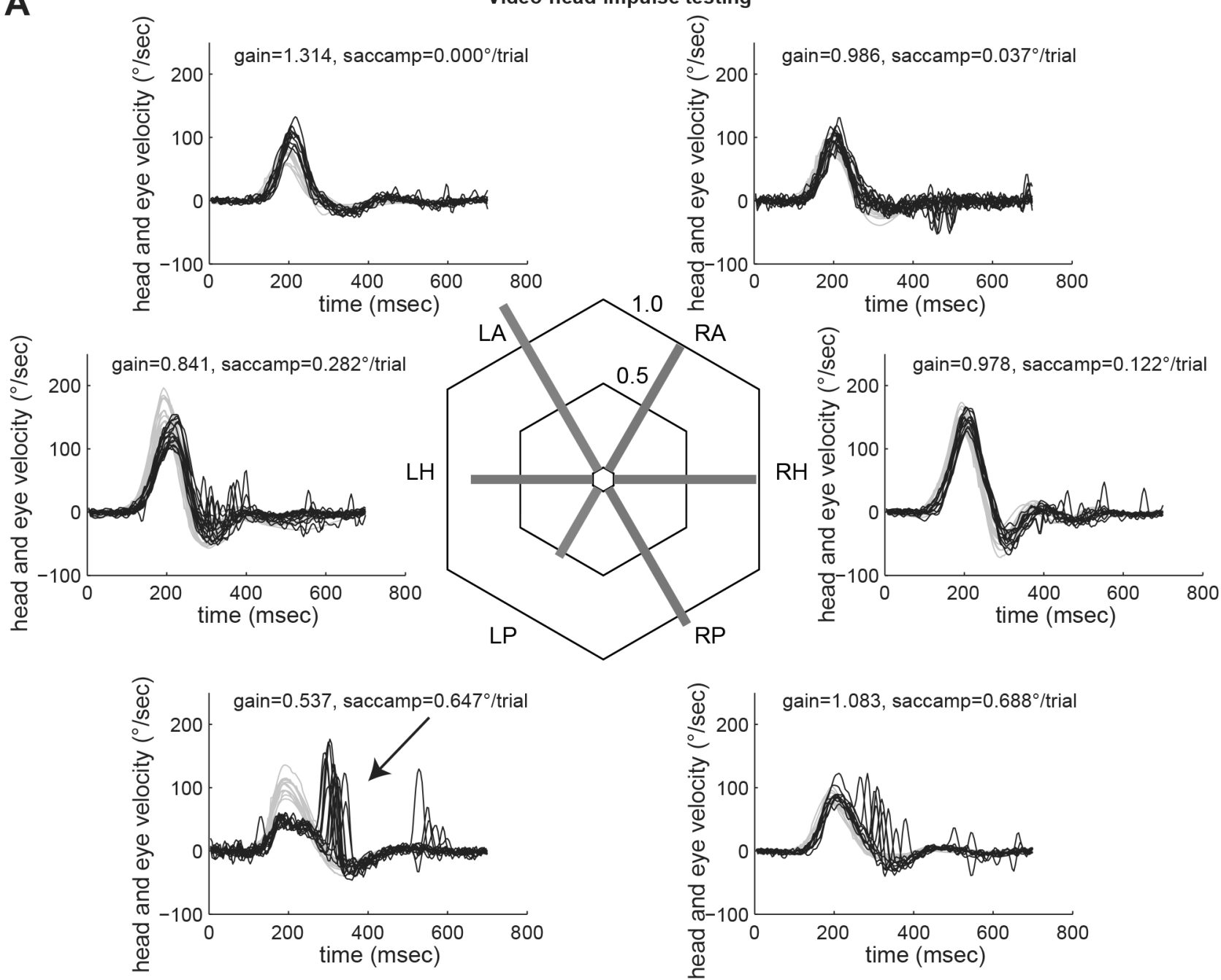

B

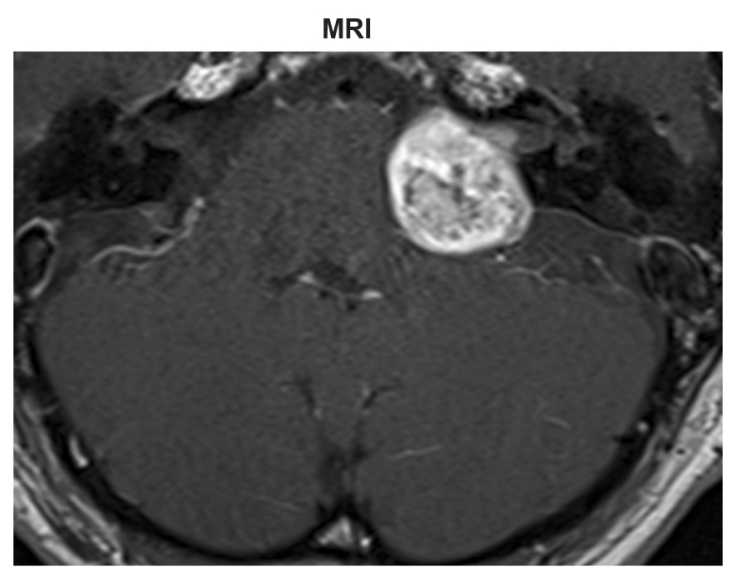

D

bone-conducted cVEMPs: AR=51\%
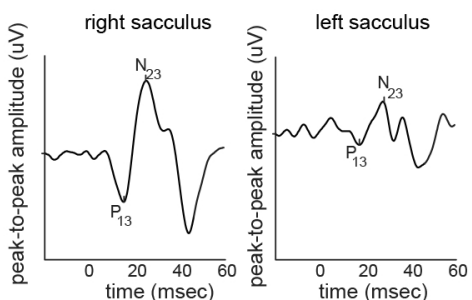

E
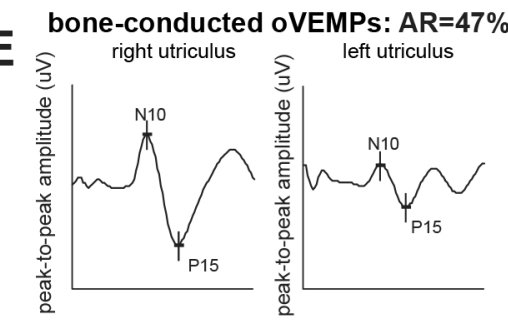

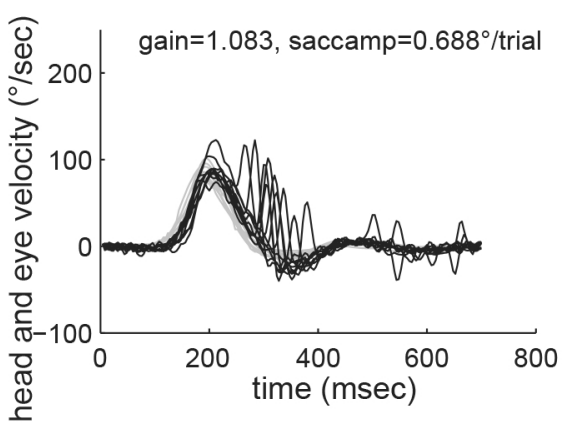

C caloric irrigation: response $44 \%$ weaker on left side

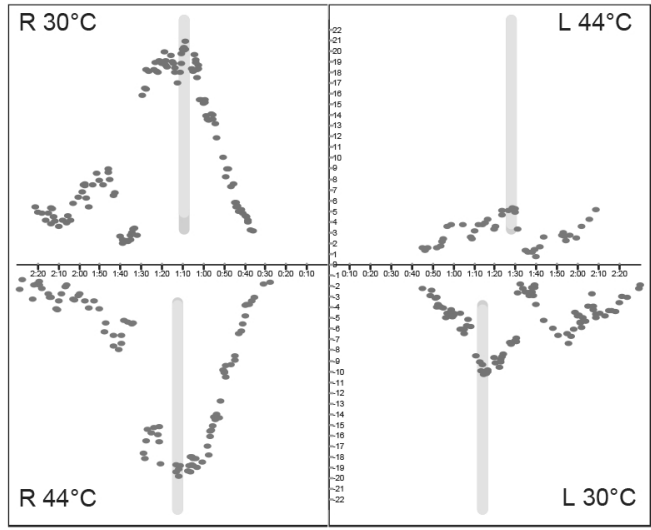

F

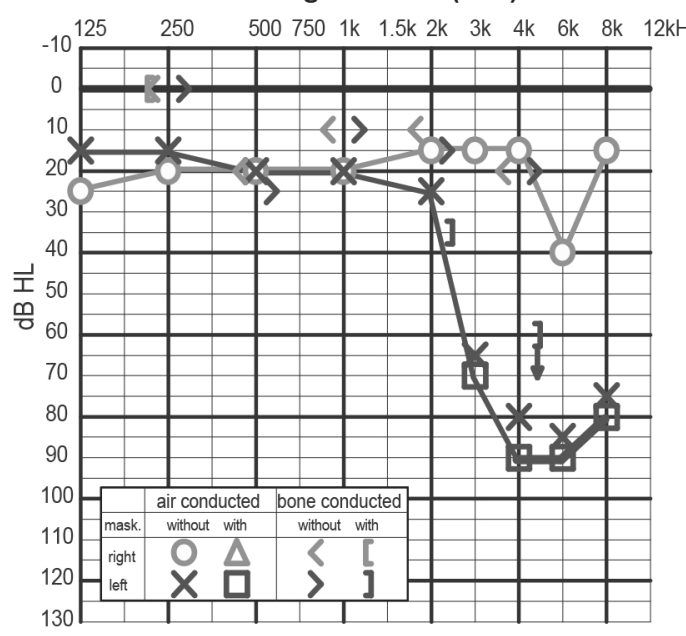




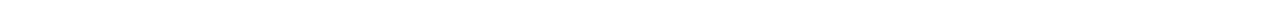




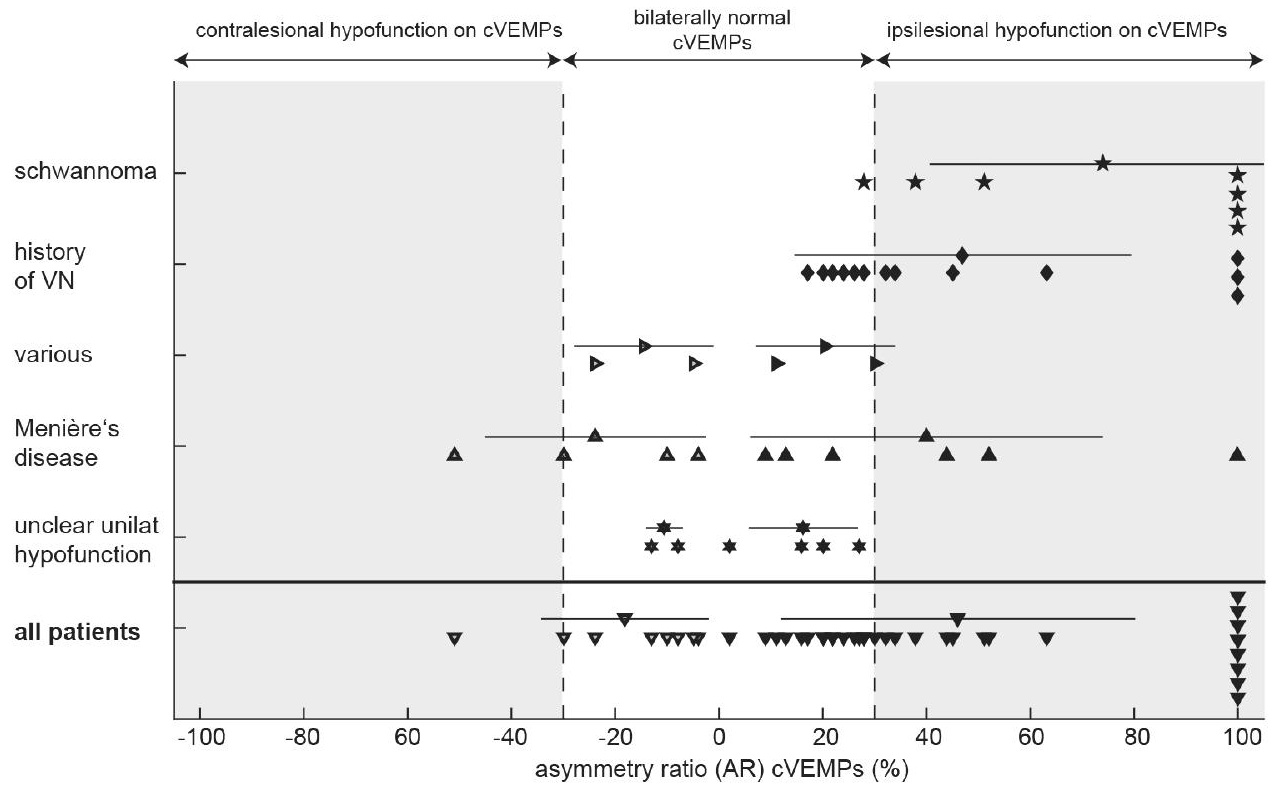


ipsilesional hearing loss (CPT-AMA $>20)$

ipsilesional hypofunction horizontal SCC (AR caloric irrigation $>25 \%$ ) ipsilesional hypofunction sacculus (AR cVEMPs $>30 \%$ )

ipsilesional hypofunction utriculus (AR oVEMPs $>30 \%$ )
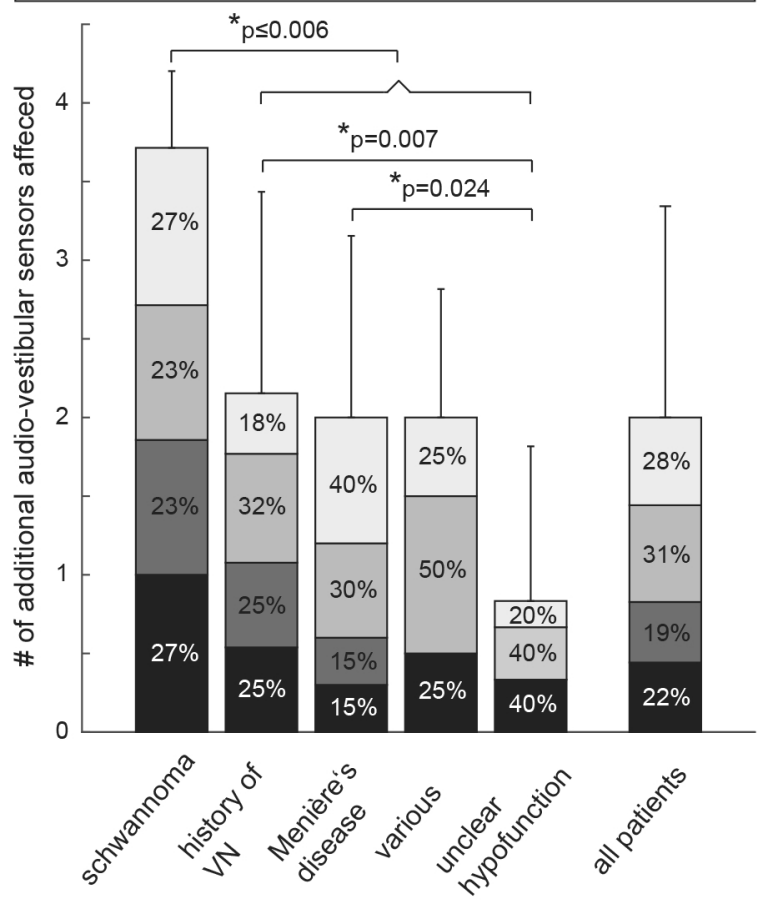